\title{
Asymmetric distribution of reconnection jet fronts in the Jovian nightside magnetosphere
}

\author{
S. Kasahara, ${ }^{1}$ E. A. Kronberg, ${ }^{2}$ T. Kimura, ${ }^{1}$ C. Tao, ${ }^{1}$ S. V. Badman, ${ }^{1}$ A. Masters, ${ }^{1}$ \\ A. Retinò, ${ }^{3}$ N. Krupp, ${ }^{2}$ and M. Fujimoto ${ }^{1}$ \\ Received 13 July 2012; revised 17 November 2012; accepted 2 December 2012; published 31 January 2013.
}

[1] Magnetic reconnection plays important roles in mass transport and energy conversion in planetary magnetospheres. It is considered that transient reconnection causes localized auroral arcs or spots in the Jovian magnetosphere, by analogy to the case in the Earth's magnetosphere. However, the local structures of transient reconnection events (i.e., magnetospheric plasma parameters) and their spatial distribution have not been extensively investigated for the Jovian magnetosphere. Here we examine plasma velocity and density during strong north-south magnetic field events in the Jovian nightside magnetosphere, which may be associated with tail reconnection. We find prominent reconnection jet fronts predominantly on the dawnside of the nightside magnetosphere, which would be a signature unique to rotation-dominant planetary magnetospheres. The observed plasma structures are consistent with significant field-aligned currents which would generate localized aurora.

Citation: Kasahara, S., E. A. Kronberg, T. Kimura, C. Tao, S. V. Badman, A. Masters, A. Retinò, N. Krupp, and M. Fujimoto (2013), Asymmetric distribution of reconnection jet fronts in the Jovian nightside magnetosphere, J. Geophys. Res. Space Physics, 118, 375-384, doi:10.1029/2012JA018130.

\section{Introduction}

[2] Transient auroral arcs and spots have been found in the Jovian ionosphere via ultraviolet and infrared observations [Grodent et al., 2003, 2004; Radioti et al., 2011]. These localized emissions appear in the nightside through dawnside and sub-corotate with the surface of the planet. The duration of these phenomena is a few to tens of minutes, or more. These signatures have been considered to be related with magnetic reconnection in the Jovian magnetosphere by analogy to the Earth's case [Radioti et al., 2010].

[3] Jovian tail reconnection has been observed in earlier work using Voyager flyby data [Nishida, 1983] and been investigated more extensively with energetic particle data from the orbiting spacecraft Galileo [e.g., Woch et al., 2002]. Kronberg et al. [2005, 2012] reported possible reconnection events (reconfiguration events), [see also Kronberg et al., 2007, 2008], which are found predominantly in the dawnside outer magnetosphere. The detailed case study on a dawnside event by Kasahara et al. [2011] illustrated plasma structures during the anomalously large southward

\footnotetext{
${ }^{1}$ Institute of Space and Astronautical Science, Japan Aerospace Exploration Agency, Sagamihara, Kanagawa, Japan.

${ }^{2}$ Max-Planck-Institut für Sonnensystemforschung, Katlenburg-Lindau, Germany.

${ }^{3}$ Laboratoire de Physique des Plasmas, CNRS, Saint-Maur-Des-Fossés, France.

Corresponding author: S. Kasahara, Institute of Space and Astronautical Science, Japan Aerospace Exploration Agency, Yoshinodai 3-1-1, Chuo-ku, Sagamihara, Kanagawa, 252-5210, Japan. (kshr@stp.isas.jaxa.jp)

(C)2012. American Geophysical Union. All Rights Reserved. 2169-9380/13/2012JA018130
}

magnetic field event reported by Russell et al. [1998]. The observed signatures were similar to the planetward-propagating "dipolarization front" seen in the Earth's magnetosphere, which is interpreted as a reconnection jet front (the leading edge of the transient reconnection jet, which is manifested as an abrupt rise in the north-south component of the magnetic field and plasma density depletion with an enhanced plasma flow [Runov et al., 2009, 2011; Sitnov et al., 2009]) and considered to have a causal link with auroral emissions [e.g., Zhang et al., 2011; Lyons et al., 2012]. A statistical analysis illustrated that such structures in the Earth's magnetosphere are found in the central plasma sheet without apparent dawn-dusk asymmetry, with an occurrence rate close to that of substorms [Fu et al., 2012]. On the other hand, the spatial distribution of jet front events in the Jovian magnetosphere has not been addressed, although energetic particle burst events, which indicate transient reconnection occurrence, have preferentially been observed on the dawnside, at least at $<120 \mathrm{R}_{J}$ [Krupp et al., 2001; Woch et al., 2002; Kronberg et al., 2005, 2012].

[4] The recent study by Vogt et al. [2010] relies more on the magnetic field signature (i.e., enhancement of the north-south component of the Jovian magnetic field: $B_{\theta}$ ), assuming southward events $\left(B_{\theta}>0\right)$ correspond to the outflow region planetward of the $X$ line and northward ones $\left(B_{\theta}<0\right)$ correspond to tailward of the $X$ line. In their study, they showed a trend that inward and outward anisotropies of energetic particles correspond to southward and northward $B_{\theta}$, respectively. Nevertheless, they revealed a large number of enhanced $B_{\theta}$ events on the duskside, in contrast to the energetic particle studies mentioned above. Therefore it is of interest to examine the energetic particle/plasma signatures, along with the list of the $B_{\theta}$ events. Keeping such a 
dawn-dusk asymmetry issue in mind, here we investigate the spatial distribution of jet fronts in the Jovian magnetosphere by combining the magnetic field, flow, and density data from Galileo.

\section{Instrumentation}

[5] In this paper we examine large $\left|B_{\theta}\right|$ events using Galileo data. The magnetic field data are obtained by the Galileo magnetometer (MAG) [Kivelson et al., 1992]. The magnetic field vector components in system-III coordinates form the standard right-handed spherical triad $(r, \theta, \phi) . B_{r}$ is along the Jupiter-to-spacecraft line, positive away from Jupiter. $B_{\phi}$ is parallel to the Jovigraphic equator (positive in the corotation direction), and $B_{\theta}$ completes the right-handed set (positive southward). The electron density is deduced from cutoff of the radio wave continuum [e.g., Kurth, 1992], which is acquired by the plasma wave subsystem (PWS) [Gurnett et al., 1992]. Ion flows are derived from the energetic particle anisotropy obtained by Energetic Particle Detector (EPD) [Williams et al., 1992].

[6] In order to quantify the anisotropy, the 16-sector flux data are fitted with the function:

$$
\begin{aligned}
J(E, \alpha, \beta)= & A_{00}(E)+A_{10}(E) \cos \alpha+A_{11}(E) \sin \alpha \cos \beta \\
& +A_{1-1}(E) \sin \alpha \sin \beta .
\end{aligned}
$$

where $E, \alpha$, and $\beta$ are the particle energy and polar and azimuthal angles in the velocity space, respectively. Coefficients $A_{n m}(E)$ are referred to as the $n$ th-order anisotropies. In this study, we calculate the zeroth- and first-order anisotropies: $A_{00}, A_{1-1}, A_{10}$, and $A_{11}$. The first-order anisotropies are converted into the JSE (Jupiter Solar Equatorial) coordinate system, resulting in $\left(A_{1 x}, A_{1 y}, A_{1 z}\right)$ or $\left(A_{1 \rho}, A_{1 \phi}, A_{1 z}\right)$; the latter is in the cylindrical coordinate expression.

[7] When the flow velocity $V$ is much lower than the detected particle velocity $v$ and the anisotropy is purely due to the bulk flow (i.e., the contributions from the pressure gradient and pitch angle anisotropy are negligible), the flow vector $\mathbf{V}$ can be calculated by

$$
\mathbf{V}=\frac{v}{2(1+\gamma)} \mathbf{A}_{1},
$$

where $\mathbf{A}_{1}=\left(A_{1 x}, A_{1 y}, A_{1 z}\right), v$ is the particle velocity of the corresponding energy channel, $\gamma=-d(\log J) / d(\log E)$ is the spectral index around the corresponding energy channel [Forman, 1970; Birmingham and Northrop, 1979]. The above equation for the flow velocity can be applied to each species and each energy channel of EPD, which allows a consistency check for the assumptions. In this study we obtain the average ion velocity as well as the standard error from several energy/species channels (two proton channels, two oxygen channels, and one sulfur channel). When the ion flow is due to the $\mathbf{E} \times \mathbf{B}$ drift, the obtained velocity should be the same for all energy/species channels, ideally. Possible causes of a large error are the pressure gradient and pitch angle anisotropy, as mentioned above.

\section{Categorization of $B_{\theta}$ events}

[8] The gray curves in Figure 1a show Galileo's orbit on the nightside when magnetic field data are available and the time resolution is $60 \mathrm{~s} / \mathrm{sample}$ or better. Circles on the trajectories indicate Galileo events listed in the previous work [Vogt et al., 2010], in which $\left|B_{\theta}\right|$ is significantly enhanced. The size of circle illustrates the peak $B_{\theta}$ value of each event, whereas the color shows the sign of $B_{\theta}$ peak (red for $B_{\theta}>0$ and blue for $B_{\theta}<0$ ). The "bipolar" events identified in the analysis by Vogt et al. [2010], for which $B_{\theta}$ is neither positive nor negative for more than $85 \%$ of the event duration, are also categorized as either positive or negative according to the sign of $B_{\theta}$ at $\left|B_{\theta}\right|$ local maximum.

[9] As can be seen in Figure 1a, the spatial coverage in the nightside magnetosphere is not uniform. Nevertheless, for $30-120 \mathrm{R}_{J}$, inside which most events occurred (see Figure 1a), the total observation times (in which the above criteria are met) are 137 days and 93 days for dawnside (local time $>1 \mathrm{~h}$ ) and duskside (local time $<23 \mathrm{~h}$ ), respectively (the difference of the observation time is $\sim 50 \%$ ). The total observation time in the midnight region (local time $=0 \pm 1 \mathrm{~h}$ ) for $30-120 \mathrm{R}_{J}$ is 70 days.

\subsection{Prominent Events}

[10] Looking at events in Figure 1a, we found that the distribution of large circles is not uniform. It can be more clearly recognized in Figure 1b, in which we plotted only prominent events (peak $\left|B_{\theta}\right|>8 \mathrm{nT}$ ). We categorized these prominent events (36 events in total) into three groups: dawnside tailward ( $B_{\theta}$-negative) events, dawnside Jupiterward ( $B_{\theta}$-positive) events, and duskside/midnight $\left(B_{\theta^{-}}\right.$ positive) events. Ion flows at the time of the $\left|B_{\theta}\right|$ peak are also displayed for prominent events (for 9 of the 36 prominent events, energetic particle data are not available) in Figure $1 \mathrm{~b}$. The dawnside $B_{\theta}$-negative events show significant tailward flows $\left(\sim 300 \mathrm{~km} / \mathrm{s}\right.$ or more), whereas the dawnside $B_{\theta^{-}}$ positive events exhibit sunward (or corotation-direction) flows. These flows (especially for $B_{\theta}$-negative events) are generally more intense and deflected compared to the average direction, which is almost azimuthal (cf. Krupp et al. [2001]). On the other hand, the duskside events show moderate flows (typically $\sim 100 \mathrm{~km} / \mathrm{s}$ ) mainly in the corotation direction (similarly to quiet times) and thus have tailward components, which is opposite to the sunward flow expected for reconnection tailward of the spacecraft location. In contrast to the dawnside, prominent events on the duskside are found only inside a radial distance of $60 \mathrm{R}_{J}$. Furthermore, prominent $B_{\theta}$-negative events are not seen on the duskside.

[11] In order to investigate the differences between these regions in more detail, first we compare example cases. In Figure 2, the three columns are for, from left to right, a dawnside $B_{\theta}$-negative event on 28 September 1996, a dawnside $B_{\theta}$-positive event on 01 April 1997, and a duskside event on 21 September 1997. For each event, we display, from top to bottom, $B_{\theta}, B_{r}$, and $B_{\phi}, v_{\perp \rho}$ (radial component of the velocity perpendicular to the magnetic field, positive outward) by solid lines and $v_{\perp \phi}$ (azimuthal component, positive in the corotation direction) by dashed lines, and the electron density by white lines plotted over the color diagram indicating radio wave intensity.

[12] The $B_{\theta}$-negative event (Figure $2 \mathrm{a}$ ) show a $B_{\theta}$ profile typical of a jet front passage (moving tailward), that is, a small positive excursion and a subsequent steep fall, followed by a gradual decay [Ohtani et al., 2004; Sitnov et al., 2009]. The magnetic signature is accompanied by an 


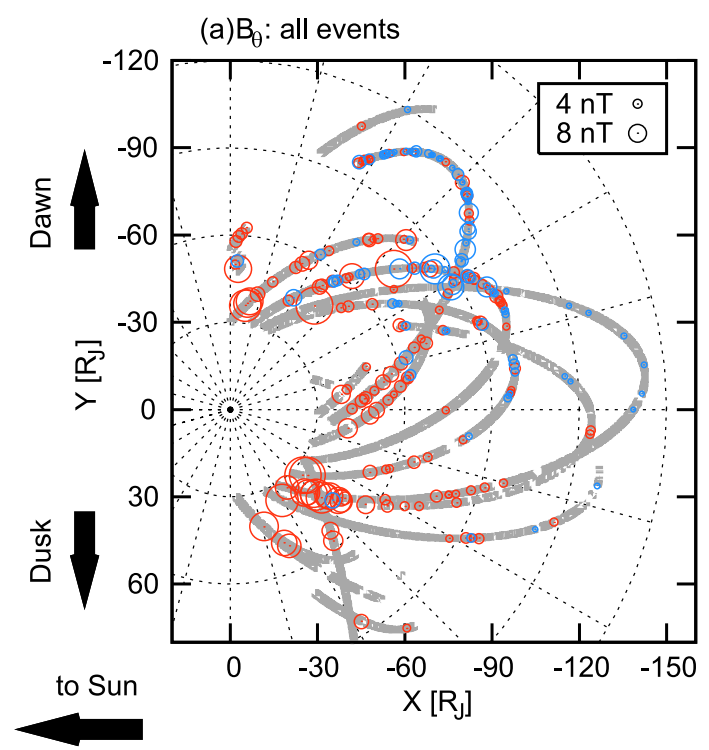

(b) $\mathrm{B}_{\theta}$ and ion flow: prominent events
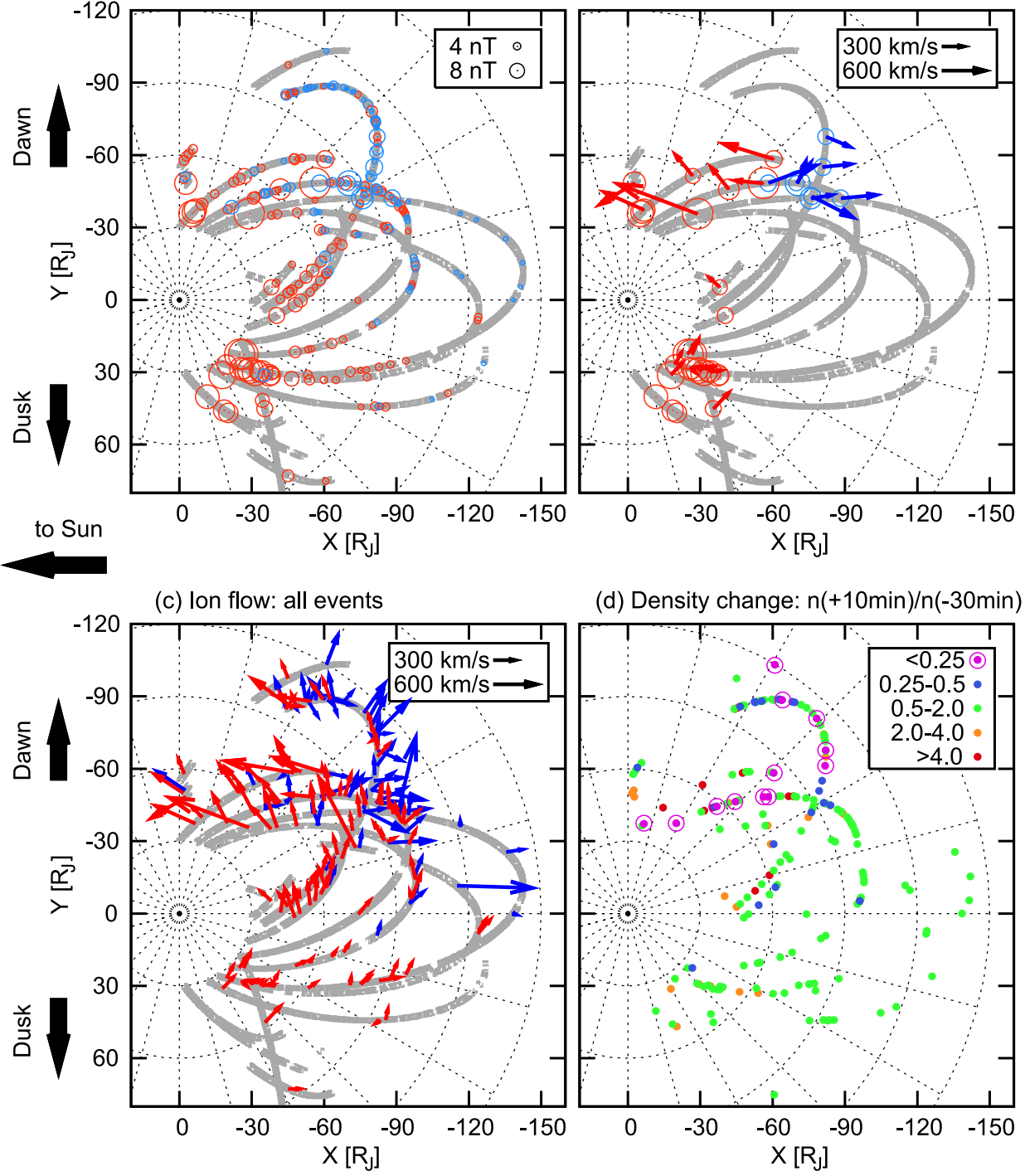

(d) Density change: $\mathrm{n}(+10 \mathrm{~min}) / \mathrm{n}(-30 \mathrm{~min})$

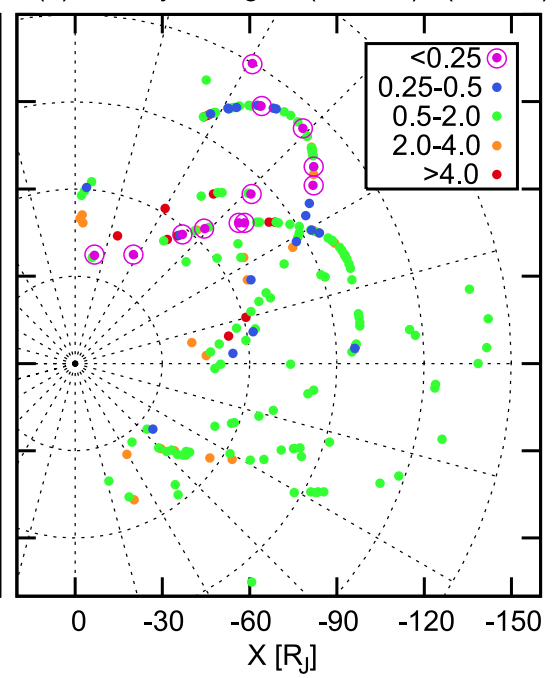

Figure 1. (a) Distribution of $B_{\theta}$ events. The size of circle indicates the peak strength of $B_{\theta}$ (reference size is indicated at the top right). Red and blue indicate those events with $B_{\theta}>0$ (southward) and $B_{\theta}<0$ (northward), respectively. (b) Same as Figure 1a but only for prominent $B_{\theta}$ events $\left(B_{\theta}>8 \mathrm{nT}\right.$ ). Ion flows perpendicular to the magnetic field are also plotted when they are available. The length of arrow displays the flow speed and reference length is indicated at the top right. (c) Ion flows perpendicular to the magnetic field for all events. (d) Density ratio between $10 \mathrm{~min}$ after the $B_{\theta}$ peak and $30 \mathrm{~min}$ before that. The value below unity indicates the density decrease after the $B_{\theta}$ peak, and vice versa. In Figures $1 \mathrm{a}-1 \mathrm{c}$, the Galileo trajectory is shown in gray only for the period in which magnetic field data with sufficient time resolution $(60 \mathrm{~s} / \mathrm{sample}$ or better) are available.

increase of the outward flow (second panel, solid line). A reversal of the azimuthal component of the flow (to anticorotation) is also seen (second panel, dashed line), reflecting the tailward deflection of the flow at this local time $(2.6 \mathrm{~h})$. Another important feature is the significant decrease of the electron density that coincides with the magnetic front passage (third panel), in conformity with typical jet front events.

[13] In the $B_{\theta}$-positive dawnside event (Figure 2b), the observed variations are consistent with a jet front planetward of the $X$ line. $B_{\theta}$ rises sharply after a small negative dip, and then decays slowly (top panel). The radial component of the ion flow stays moderate (second panel, solid line) whereas the azimuthal component enhances (second panel, dashed line), indicating the sunward flow intensification. The electron density abruptly decreases at the $B_{\theta}$ front. These features are consistent with a propagating jet front sunward of an X line [Ohtani et al., 2004; Sitnov et al., 2009; Runov et al., 2011; Fu et al., 2012].

[14] The dusk event (Figure 2c) is somewhat different to the above two cases. The baseline of $B_{\theta}$ is rather high $(>5 \mathrm{nT})$ compared to the dawnside events, and hence sharp increases are not clearly seen. Although the radial component of the ion flow (second panel, solid line) shows a slight 

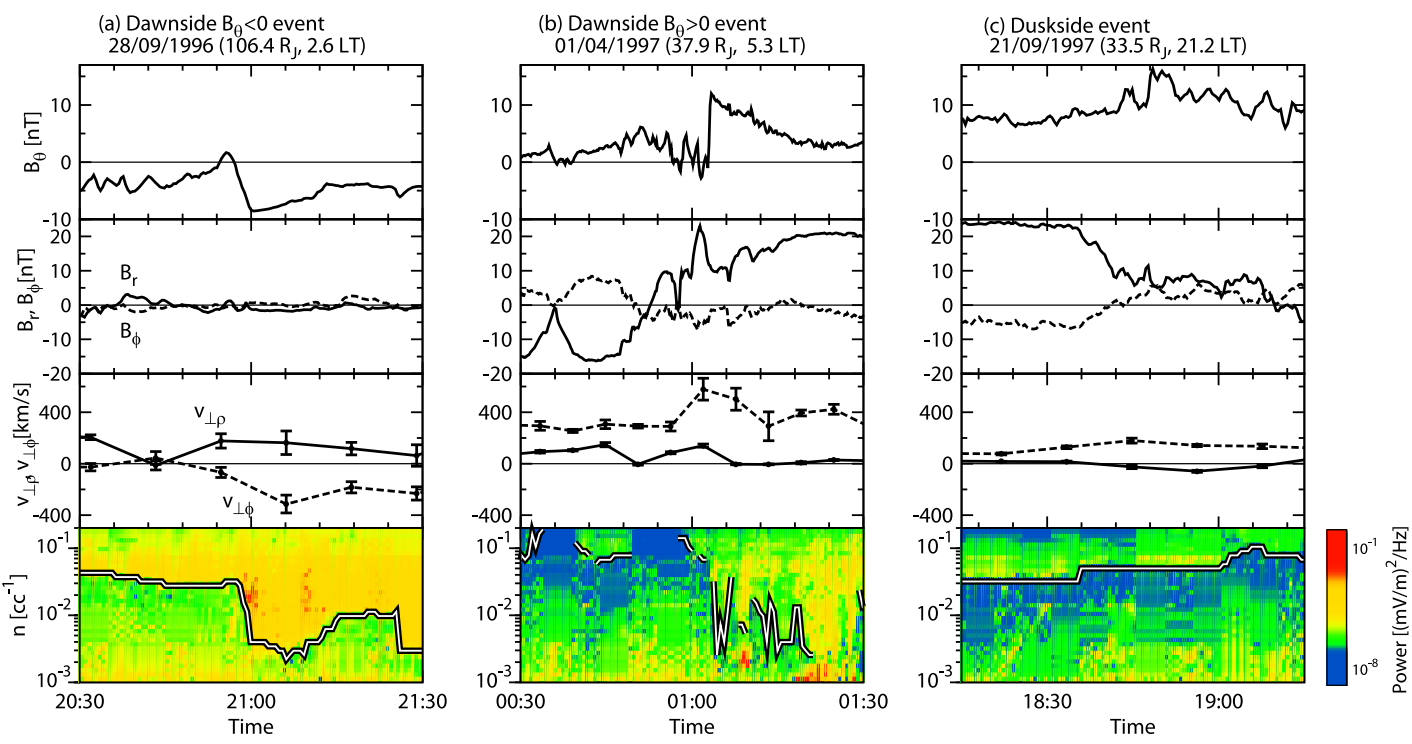

Figure 2. Typical examples of prominent events. From left to right, (a) dawnside $B_{\theta}$-negative event, (b) dawnside $B_{\theta}$-positive event, and (c) duskside event are displayed. First row shows the $B_{\theta}$ time profile, the second row shows the radial and azimuthal components of the magnetic field $\left(B_{r}\right.$, solid lines, and $B_{\phi}$, dashed lines, respectively), the third row shows the radial $\left(v_{\perp \rho}\right.$, solid lines) and azimuthal $\left(v_{\perp \phi}\right.$, dashed lines) components of plasma flow perpendicular to the magnetic field, and the bottom panels show the electron density by white lines. Frequency-time spectrograms of the radio wave (color indicates the wave power) are also plotted in the bottom panel, with the frequency $f$ being converted into the density $n$ by the equation $n=(f[\mathrm{~Hz}] / 8980)^{2}\left[\mathrm{~cm}^{-1}\right]$.

decrease (i.e., became slightly planetward), the azimuthal component increased (second panel, dashed line) and the flow direction remains in the corotation direction. Furthermore, the electron density is constant across the $B_{\theta}$ peak, in contrast to the dawnside cases.

[15] The three events displayed above are representatives of the three categories (i.e., dawnside $B_{\theta}$-negative, dawnside $B_{\theta}$-positive, and duskside), and thus most of the above signatures can be applied to other prominent events in each category. In order to illustrate that, we conducted superposed epoch analyses on the prominent events (Figure 3 ). The reference time $(t=0)$ is defined to be the peak of $\left|B_{\theta}\right|$. The three columns of Figure 3 are for, from left to right, dawn $B_{\theta}$-negative events ( 8 cases), dawn $B_{\theta}$-positive events ( 9 cases), and dusk/midnight events ( 19 cases, $B_{\theta}>0$ for all of them). Here we include midnight events (two events, at local times of 23.4 and $0.5 \mathrm{~h}$ ) in the dusk category since the time profile of the parameters show the same trend as the dusk events. The average values and standard deviations are represented by thick black lines and gray shades, respectively. The time profiles for each event are also displayed by the colored lines. Several velocity data points with a large error (when the relative error is $>50 \%$ and also the absolute error is $>150 \mathrm{~km} / \mathrm{s}$ for dawnside cases and $>50 \mathrm{~km} / \mathrm{s}$ for duskside cases) are excluded from the plots and superposed epoch averaging, although such exclusion does not cause any substantial change in the average profile (note also that data points around the $B_{\theta}$-peak, which are shown in Figure $1 \mathrm{~b}$, do not show such a large error). The reliability of the density data has also been visually checked event by event (i.e., lines in the fourth panel are drawn only when the radio continuum cutoff is visible). In addition to $B_{\theta}$, flow, and electron density, here we also display the magnetic bendback angle $\alpha \equiv \arctan \left(B_{\phi} / B_{r}\right)$ (plotted only when $\left|B_{r}\right|>1 \mathrm{nT}$ to exclude the very center of the current sheet) at the bottom, in order to examine any trend of equatorial current structures for each category. When the equatorial current is radially outward, we expect $\alpha<0$.

[16] For the $B_{\theta}$-negative events (in the first column of Figure 3 ), the $B_{\theta}$ profiles are the same as the case in Figure $2 \mathrm{a}$ (the first panel, a steep fall followed by a gradual decay). Most events have positive excursions before the abrupt negative rise, although it is not seen in the averaged profile (black thick line) due to the differences in the timings of the positive small peaks. The enhanced outward flows (second panel) associate with the $B_{\theta}$ change in most events. Not always but in several cases, decreases of the azimuthal velocity of the flow are also seen (third panel). The decrease of the electron density is observed as a general trend (fourth panel). Although the averaged $\alpha$ shows some variation, the deviation is quite large and it is hard to unambiguously deduce a clear trend for this category.

[17] For the $B_{\theta}$-positive dawnside events shown in the second column of Figure 3, the same trends as shown in Figure $2 \mathrm{~b}$ are seen. $B_{\theta}$ rises sharply and then decays slowly (top panel). Negative dips before the $B_{\theta}$ rises are observed in about half of events, although it is not clear in the average profile. The radial component of the ion flow on average stays moderate (second panel) whereas the standard deviation becomes larger. The azimuthal component of the velocity enhances (third panel), and the electron density decreases in most cases. Before the $B_{\theta}$ peak, $\alpha$ is negative on average, in conformity with the usual swept-back magnetic field configuration (corresponding to radially outward near-equatorial 

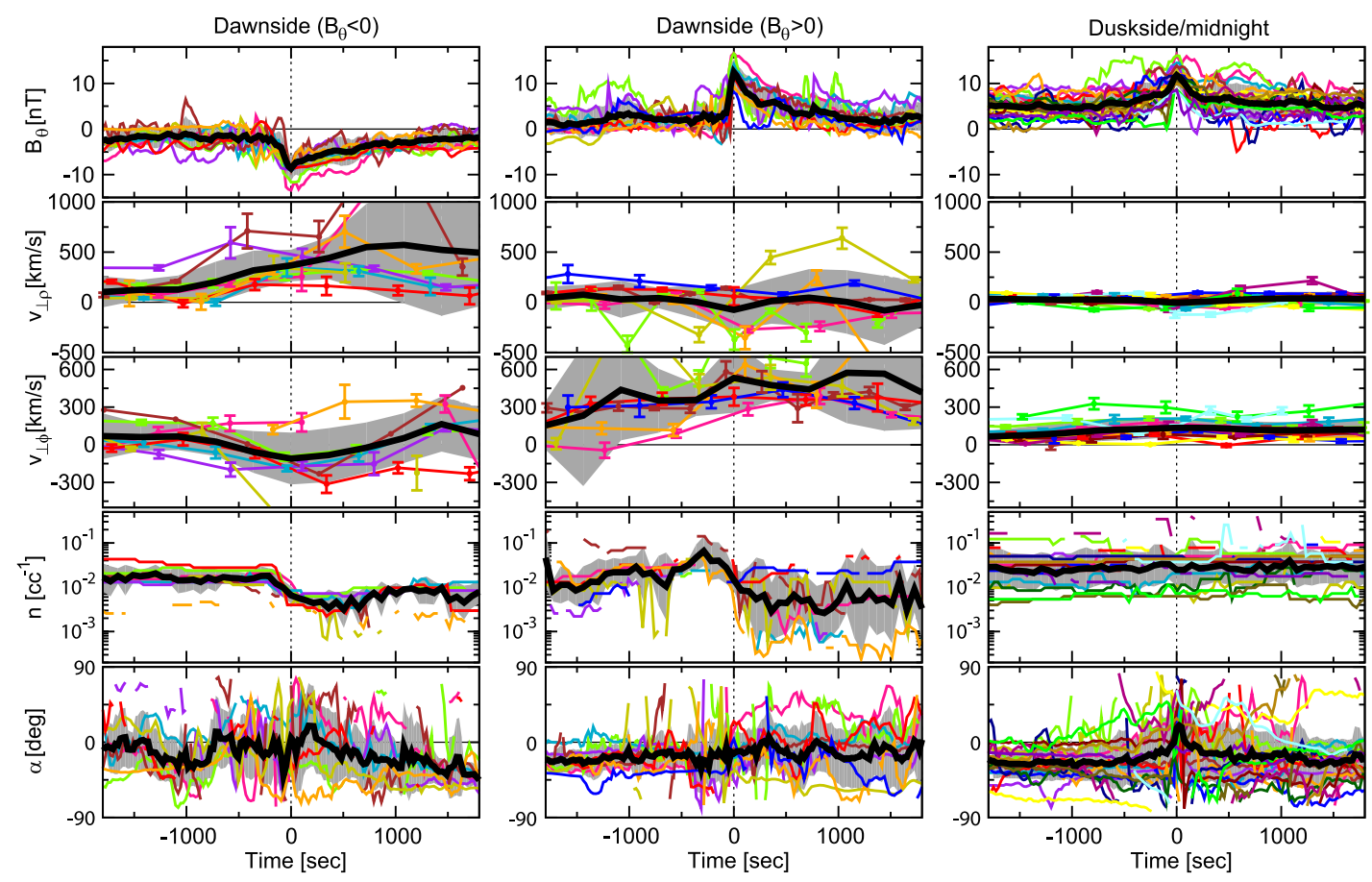

Figure 3. Superposed epoch plots of plasma and field parameters for each category. From top to bottom, $B_{\theta}, v_{\perp \rho}, v_{\perp \phi}$, the electron density $n$, and magnetic bend-back angle $\alpha \equiv \arctan \left(B_{\phi} / B_{r}\right)$ are shown. The average values and standard deviations are shown by thick black lines and gray shades, respectively. Each colored line corresponds to a particular event throughout a column.

current). The deviation becomes large after the $B_{\theta}$ peak, and $\alpha$ even becomes positive in some cases, indicating the reduction (or sometimes reversal) of the radial tail current in the plasma sheet.

[18] In sharp contrast to the previous two categories, the dusk/midnight events in the third column of Figure 3 do not show jet front signatures. The average $B_{\theta}$ rises gradually and then decreases, showing an almost symmetrical time profile about the reference time $(t=0)$. The ion radial flow very slightly decreases at the $B_{\theta}$-peak on average (the second panel), while the azimuthal flow tends to increase. The average electron density is constant, the same as the case in Figure 2c. The bottom panel illustrates the large deviation of $\alpha$ over the whole period. A significant variation is the increase of $\alpha$ around the $B_{\theta}$ peak, which indicates the reduced (or reversed) radial current near the equator. Note also that, for the dusk/midnight events, the baseline level is $\sim 5 \mathrm{nT}$ on average, which is higher than those for the dawnside events (approximately $-2 \mathrm{nT}$ for negative events and $\sim 2 \mathrm{nT}$ for positive events). Due to this difference in the offset, the duskside/midnight category includes less prominent events in terms of the difference in the height between the $B_{\theta}$-peak and the baseline. Nevertheless, such an effect is not substantial in our study, since the characteristics of the ion flow and density are very similar in the right panels of Figure 3, irrespective of the $B_{\theta}$ values.

\subsection{Moderate Events}

[19] In the previous subsection we have mainly focused on prominent events (peak $\left|B_{\theta}\right|>8 \mathrm{nT}$ ), which clearly indicate the preferential appearance of reconnection jet fronts on the dawnside. Moderate events (peak $\left|B_{\theta}\right| \leq 8 \mathrm{nT}$ ), however, are more complex. They show a variety of magnetic/plasma signatures, some of which are similar to the prominent events and some are not. Hence, in order to avoid mixing different kinds of phenomena, we do not attempt superposed epoch analyses for moderate events, and instead we show several examples in Figure 4.

[20] Some of the moderate dawnside events show jet front events which are similar to the prominent events (e.g., Figure 4a), but there are many events which do not exhibit sharp changes in $B_{\theta}$ and the density (e.g., Figure $4 \mathrm{~b}$ ).

[21] Most of the moderate duskside/midnight events show the same signatures as the prominent duskside/midnight events (i.e., moderate corotational flow and constant density). Indeed, Figure 1c illustrates predominant corotation flows on the duskside/midnight (events with a large error are excluded, similarly to the superposed epoch analyses above). However, several events show significant inward deflection of the flow (e.g., Figure 4c) as well as negative $B_{\theta}$ events with outward flow (e.g., Figure 4d), although such events are not the majority (note that flow data in Figure $4 \mathrm{~d}$ are only suggestive since the error of $v_{z}$ is large and thus not shown in Figure 1c). These events indicate that reconnection can also take place on the duskside. Despite such reconnection signatures, however, no substantial density decrease was seen for the duskside events, compared to typical jet front events on the dawnside. In order to demonstrate that, we plotted the density change in Figure 1d. The ratios of the density at $30 \mathrm{~min}$ before the $B_{\theta}$-peak (as a reference for preconditions) and that at $10 \mathrm{~min}$ after the $B_{\theta^{-}}$ peak (representing disturbed states) are shown by the colored circles. On the duskside, the green color dominates, illustrating a moderate density change around $B_{\theta}$-peak. In contrast, 
(a) $23 / 03 / 1997\left(77.5 R_{j}, 3.3 \mathrm{LT}\right)$
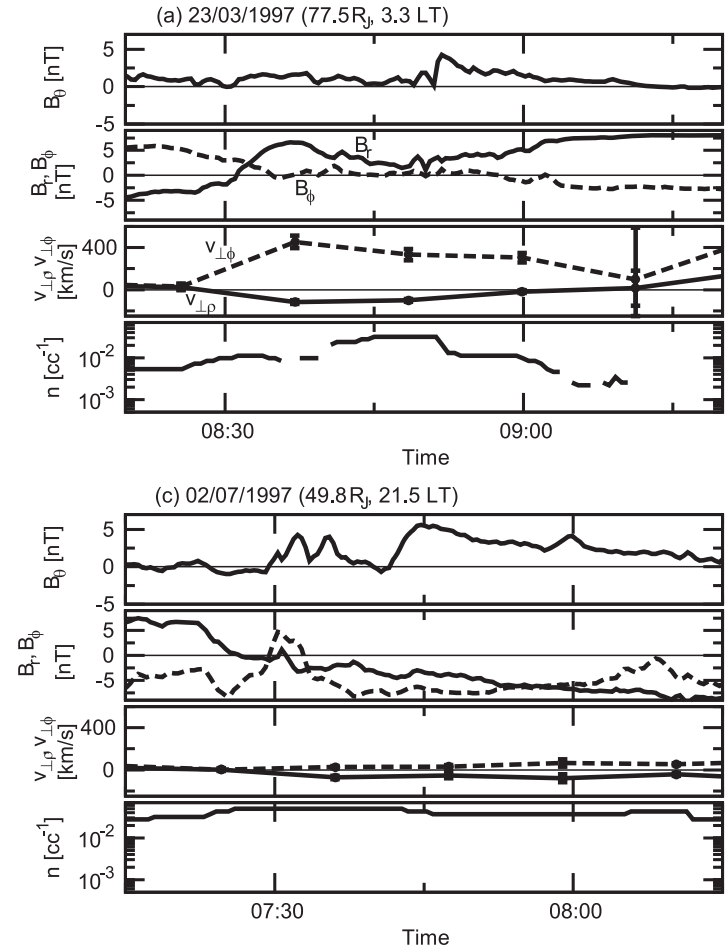

(e) $24 / 03 / 1997\left(75.3 R_{\mathrm{f}}, 3.39 \mathrm{LT}\right)$

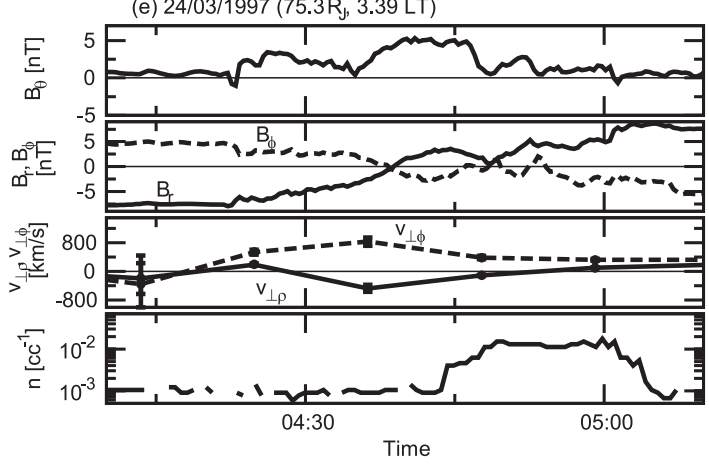

(b) $20 / 10 / 1996(98.9 R, 4.1 \mathrm{LT})$

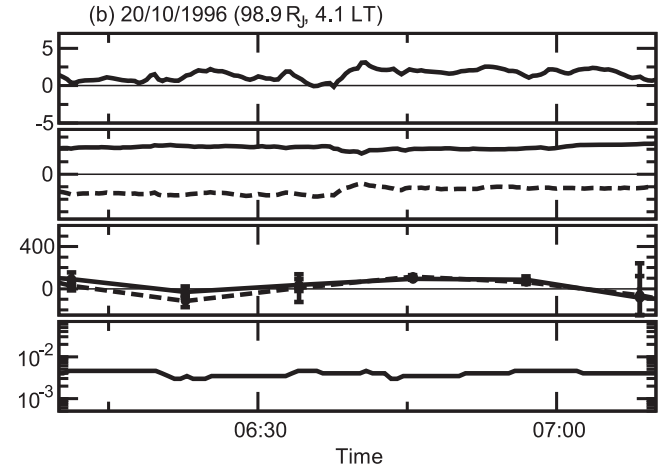

(d) $05 / 12 / 1998\left(93.8 R_{b}, 22.1 \mathrm{LT}\right)$

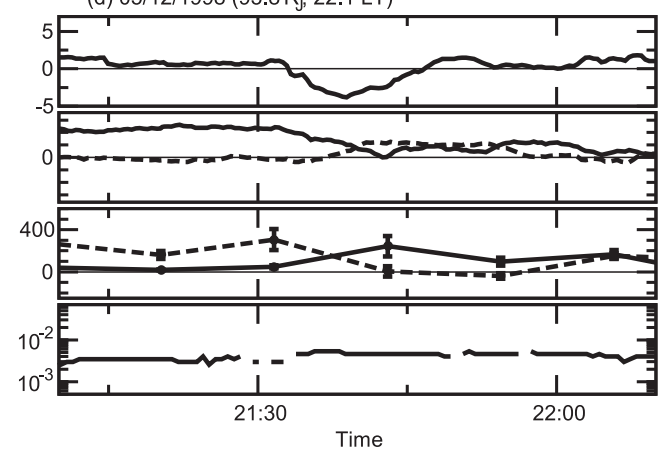

(f) $03 / 09 / 1998\left(115.6 R_{b}, 0.38 L T\right)$

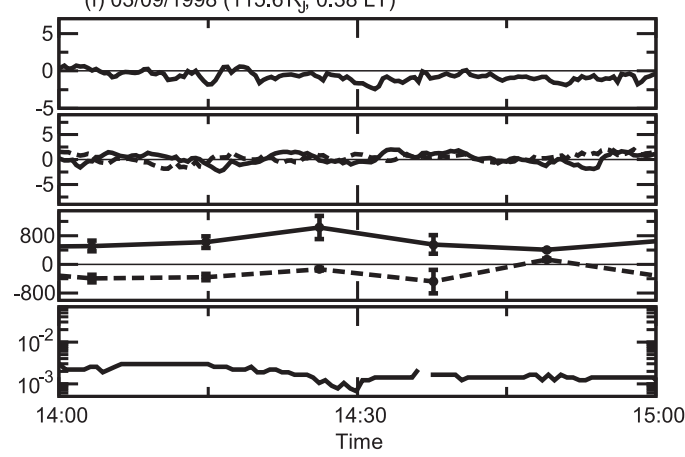

Figure 4. Examples of moderate events. $B_{\theta}$ (first row), radial ( $B_{r}$, solid) and azimuthal ( $B_{\phi}$, dashed) components of the magnetic field (second row), radial $\left(v_{\perp \rho}\right.$, solid) and azimuthal $\left(v_{\perp \phi}\right.$, dashed) components of the perpendicular velocity (third row), and the electron density (fourth row) are shown for six events. Vertical scales are the same except the velocity data in the bottom two events.

on the dawnside, not only the prominent events but also some moderate events exhibit a significant density drop after $B_{\theta}$ peak (purple color).

[22] Finally, in order to give a broader view on the different characteristics of $B_{\theta}$ events, we added two examples in Figures $4 \mathrm{e}$ and $4 \mathrm{f}$. These are categorized in moderate events according to our $B_{\theta}$ criterion, but with strong flows (note that the vertical range of the velocity data is different from Figures $4 \mathrm{a}-4 \mathrm{~d}$ ). In Figure $4 \mathrm{e}$, although the indication of the sharp increase of $B_{\theta}$ is seen, it did not reach our threshold (i.e., $8 \mathrm{nT}$ ). This seems to be attributed to the fact that the spacecraft was located far from the plasma sheet center, in conformity with the large $B_{r}$ and low density $\left(<10^{-3} \mathrm{~cm}^{-3}\right)$. Such a low density even at the center of the plasma sheet $\left(B_{r} \sim 0 \mathrm{nT}\right.$ at $\left.0438 \mathrm{UT}\right)$ suggests lobe reconnection, and then the subsequent density increase by an order of magnitude may correspond to the cessation of lobe reconnection. In another event (Figure 4f), the strong outward flow (up to $\sim 1000 \mathrm{~km} / \mathrm{s}$ ) was detected with the moderately negative $B_{\theta}$, although the magnetic field inclination was large (not explicitly shown here). In this event the spacecraft seemed to be around the center of the plasma sheet. This event tells us that the strong flow is not the sufficient condition to produce a clear jet front. This type of events (i.e., strong flow without an obvious sharp front) is common for bursty bulk flow events in the Earth's magnetosphere.

\section{Discussion}

\subsection{Dawn-Dusk Asymmetry}

[23] We have found a dawn-dusk asymmetry in the $B_{\theta^{-}}$ enhanced events. Dawnside events show striking features which are consistent with reconnection jet fronts, especially for prominent events (peak $\left|B_{\theta}\right|>8 \mathrm{nT}$ ). Some moderate events also illustrate similar signatures. Prominent $B_{\theta}$-negative events are found at $75-110 \mathrm{R}_{J}$ and prominent $B_{\theta}$-positive 
events at $35-85 \mathrm{R}_{J}$ on the dawnside. In sharp contrast, on the duskside and in the midnight region, prominent events are concentrated near Jupiter $\left(<60 \mathrm{R}_{J}\right)$, whereas total observation times are 29 days for $30-60 \mathrm{R}_{J}$ and 37 days for $60-90 \mathrm{R}_{J}$ on the duskside (14 days for $30-60 \mathrm{R}_{J}$ and 17 days for 60 $90 \mathrm{R}_{J}$ in the midnight sector). In addition, no negative prominent events are found on the duskside/midnight in spite of the significant total observation time (in the radial range of 80-150 $\mathrm{R}_{J}, 51$ days on the duskside and 99 days in the midnight region, compared to 92 days on the dawnside). Furthermore, the plasma flow is moderately sub-corotating (with a slight increase in azimuthal velocity), indicating the plasma came from duskward of the spacecraft, rather than a tail $X$ line. The prominent duskside events, therefore, may not be reconnection flow events and may be caused by another process (such as field lines transiently being stretched dusk/ tailward by the convection and then pulled back by magnetic tension [cf. Ogino et al., 1998; Walker et al., 2001]). Nevertheless, here we do not rule out duskside reconnection occurrence since several moderate events are not inconsistent with reconnection outflow signatures in terms of their $B_{\theta}$ profiles and radial flows. However, there is still a significant difference in the density profile compared to the dawnside cases. We found a tendency for dawnside events to be associated with significant density depletion, whereas duskside/midnight events typically exhibit almost constant density profiles.

[24] Such a difference may be attributed to the two different types of reconnection: plasma sheet reconnection (i.e., reconnection of closed magnetic field lines) and lobe reconnection (i.e., reconnection of open magnetic field lines). Although the closed field reconnection would lose some plasma content by pinching off plasmoids, the open field reconnection should result in more drastic density depletion in reconnected flux tubes due to the intrusion of the lobe plasma. Considering the thinner plasma sheet on the dawnside (at least inside $<150 \mathrm{R}_{J}$, where Galileo has investigated; cf. Waldrop et al. [2005]), open field reconnection is likely to occur preferentially on the dawnside, which can explain the predominant density decrease on the dawnside. A sudden increase of the reconnection outflow speed due to the initiation of lobe reconnection (note that the lower density results in a higher Alfvén velocity and thus faster outflow) would also contribute to the steepening of jet front signature. If this is the case (i.e., jet fronts are formed by the transition to lobe reconnection), the signatures observed prior to jet fronts (e.g., the moderately negative $B_{\theta}$ and the flow change well before the $\left|B_{\theta}\right|$ peak seen in the left column in Figures 2 and 3, and also the substantial change of the ion flow in Figure 4a) may correspond to plasma sheet reconnection.

[25] Preferential lobe reconnection on the dawnside is consistent with the model proposed by Cowley et al. [2003], which indicates Dungey-cycle operation on the dawnside. It should be noted, however, that some global MHD simulations [Walker et al., 2001; Fukazawa et al., 2005, 2006, 2010] illustrate that the lobe reconnection $X$ line can extend over the whole tail from dawn to dusk, when the northward interplanetary magnetic field continues for a long time $(\sim 100 \mathrm{~h})$. In such simulations, the reconnection outflow sunward of $X$ line is rather azimuthal on the duskside. Considering the limited spatial coverage of Galileo, therefore, we cannot exclude the extension of the lobe reconnection $X$ line to the duskside in the distant tail (e.g., outside $150 \mathrm{R}_{J}$ ).
[26] It is interesting here to compare our result to a global MHD simulation of the Saturnian magnetosphere [Jia et al., 2012]. Their simulation illustrates strong reconnection outflows on the dawnside accompanying mass-depleted flux tubes, in good agreement with our observations. Importantly, the significant intrusion of the low-density plasma is due to lobe reconnection, in conformity with our discussion above (unfortunately, Jovian simulation papers above do not display density snapshots in a comparable manner). Along with these results, a dawn-dusk asymmetry of the occurrence of propagating jet front events may also exist in the Saturnian magnetosphere, in contrast to the Earth's case [Fu et al., 2012].

\subsection{Jet Front Thickness and Ion Composition}

[27] Observations in the Earth's magnetosphere have determined the thickness of Earthward propagating jet fronts (dipolarization fronts). According to the literature, the front thickness is typically $300-1000 \mathrm{~km}\left(\sim 0.05-0.15 \mathrm{R}_{E}\right.$ [e.g., Sergeev et al., 2009; Runov et al., 2011; Fu et al., 2012]), corresponding to a few ion inertial lengths. The thickness can be calculated by the rise time of the north-south component of the magnetic field multiplied by the propagation speed of the front. The propagation speed can be determined by multi-spacecraft timing analyses, or assumed to be close to the local ion flow velocity. Similar results have been obtained in both cases [e.g., Runov et al., 2011; Fu et al., 2012]. Using the latter assumption, here we attempt to deduce the front thickness of the dawn jet front events. Referring to Figure 3, the rise time of $B_{\theta}$ for negative events is $\sim 70 \mathrm{~s}$ on average, whereas it is $\sim 60 \mathrm{~s}$ for positive events (the foot regions just in front of the sharp $B_{\theta}$ rises, which show more gradual rises over $>1 \mathrm{~min}$, are excluded from these rise times). The average ion flow speeds at the front are $\sim 380 \mathrm{~km} / \mathrm{s}$ and $\sim 550 \mathrm{~km} / \mathrm{s}$ (for each event, the flow direction is close to the front normal determined by the minimum-variance method, although not shown), resulting in the thicknesses of $\sim 30,000 \mathrm{~km}\left(\sim 0.4 \mathrm{R}_{J}\right)$ in both cases. This value corresponds to $\sim 3 c / \omega_{p i}$ where $c$ is the light speed, $\omega_{p i}$ is the ion plasma frequency, and thus $c / \omega_{p i}$ is the ion inertial length (here we applied $n=6 \times 10^{-3} \mathrm{~cm}^{-3}$ as the typical density around the $B_{\theta}$ peak). Note that in the above estimation we assumed a heavy-ion dominant plasma (ion mass-percharge of 10) for the calculation of $c / \omega_{p i}$ (i.e., $m_{i} / q=10 m_{p} / e$ for $\omega_{p i}=\sqrt{4 \pi n e q / m_{i}}$, where $m_{i}$ is the ion mass, $q$ is the ion charge, $e$ is the elementary charge, and $n$ is the electron density), considering $\mathrm{O}^{2+}$ and/or $\mathrm{S}^{3+}$ as the main ion species [cf. Geiss et al., 1992]. If $m_{i} / q$ is larger (e.g., due to the significant presence of $\mathrm{O}^{+}, \mathrm{S}^{2+}$, and/or $\mathrm{S}^{+}$), the ion inertial length becomes larger and the normalized front thickness becomes smaller. Conversely, if the average $m_{i} / q$ is close to $m_{p} / e$ (due to the dominant proton presence), the normalized front thickness is larger by a factor of $\sim 3$. Although the ion composition of the plasma is unclear for our events, we stress that the assumption of $m_{i} / q=10 m_{p} / e$ (or larger) gives a close result to the Earth's case, compared to $m_{i} / q=m_{p} / e$.

\subsection{Field-Aligned Current}

[28] The plasma structure of the dawn $\mathrm{B}_{\theta}$-positive events is similar to dipolarization fronts in the Earth's magnetosphere, hence enhancement of the field-aligned current and 


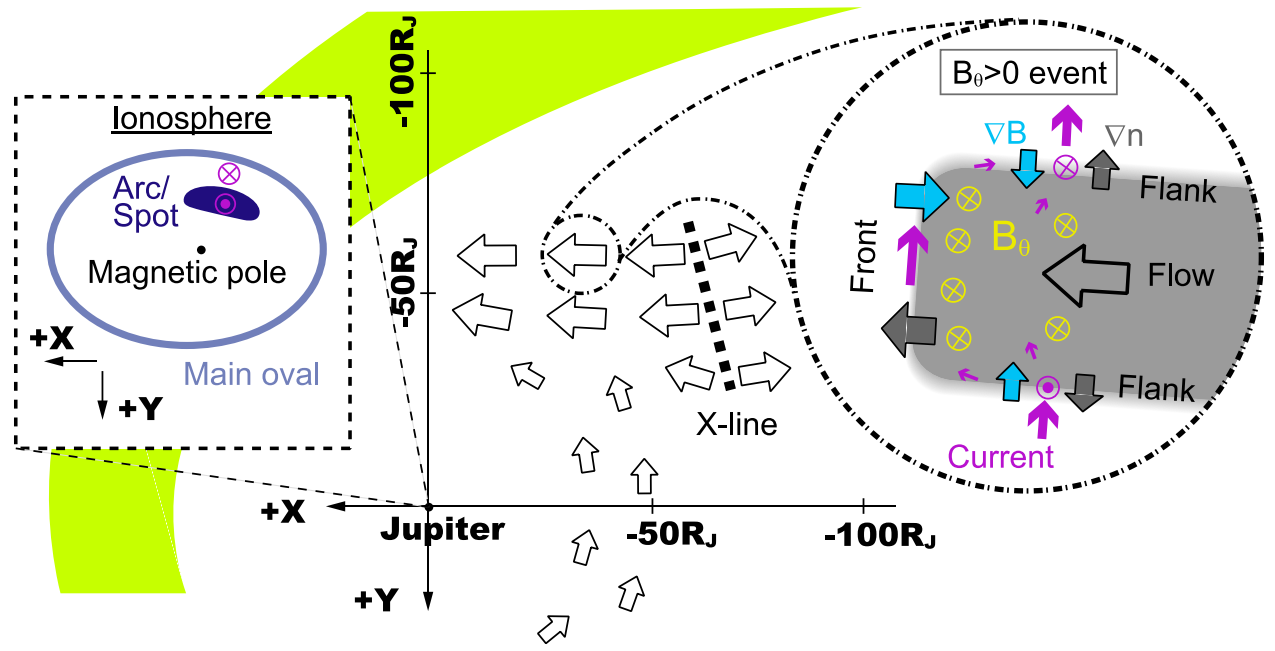

Figure 5. Schematic showing the relationship between signatures in the ionosphere and the nearequatorial magnetosphere (the northern side of magnetosphere is illustrated, and note that the out-of-plane current is reversed if the southern side is viewed from the north). Gray, blue, and purple arrows indicate density gradient, magnetic field strength gradient, and electric current, respectively. $B_{\theta}$ is indicated in yellow, and plasma flow is illustrated by blank arrows. The likely range of the magnetopause location is also displayed by a green curved shape from top to left. The ionospheric signature in the left inset is not to scale.

associated auroral illumination are expected by analogy. Figure 5 illustrates our interpretation of the observations. The radial current in the horizontal current sheet is reduced inside the flow structure, where $B_{\theta}$ and thus $|B|$ are enhanced and the plasma density is depressed, as indicated by the second column in Figure 3. A certain amount of such currents deflected at the flanks would close at the jet front, where the thin vertical current sheet corresponds to the steep $B_{\theta}$ gradient (see Zhang et al. [2011] for a schematic threedimensional view). At the same time some fraction of the tail current would reach the ionosphere, resulting in an auroral arc or spot. Such auroral signatures should appear poleward of the main oval, since the jet structures are detected outside 20-30 $\mathrm{R}_{J}$, where the main oval maps to [Cowley et al., 2005].

[29] The field-aligned current intensity can be estimated through the following equation [e.g., Hasegawa and Sato, 1979; Lui, 1996]:

$$
j_{\|}(s) \sim B(s) \int_{0}^{s}\left[\frac{2 \nabla B \cdot \mathbf{j}_{\perp}}{B^{2}}+\frac{n m_{i}}{Z B} \frac{d}{d t}\left(\frac{\Omega}{B}\right)-\frac{\nabla n \cdot \mathbf{j}_{\mathbf{i n}}}{n B}\right] d s^{\prime},
$$

where $j_{\|}$and $\mathbf{j}_{\perp}$ are the current density parallel and perpendicular to the magnetic field, respectively, $\mathbf{j}_{\text {in }}$ is inertial current, $n$ is electron density, $m_{i}$ is ion mass, $Z$ is the average charge state number of thermal ions, $B$ is the magnetic field strength, and $\Omega \equiv(\nabla \times \mathbf{v}) \cdot \mathbf{B} / B$ is vorticity (the vortex vector is assumed to be field-aligned). The integral is taken along the magnetic field line; $s$ and $s^{\prime}$ are the field-aligned distance from the equator, positive in the magnetic field direction.

[30] The contribution from the first term on the right hand side can be approximated by $j_{\| 1}(h) \sim 2 B(h)\left(\Delta B / D_{B}\right) j_{\perp} h / B_{e}^{2}$, where $h$ is the thickness of the current sheet (i.e., scale length of the assumed current deflection region), and $B(h)$ and $B_{e}$ are the magnetic field at $s=h$ and the equator, respectively, and $D_{B}$ is the spatial scale of the magnetic field variation $\triangle B$ at the flank. For an order-of-magnitude estimate, we roughly take $B(h) \sim B_{e} \sim 10 \mathrm{nT}, \Delta B \sim 10 \mathrm{nT}$ (cf. the second column of Figure 3), $D_{B} \sim 1 R_{J}$ (not well constrained from observations, but we expect it to be larger than the jet front thickness: $\sim 0.4 \mathrm{R}_{J}$ on average; see section 4.2 ), and $j_{\perp} \sim$ $B_{\text {lobe }} /\left(\mu_{0} h\right)$, where $\mu_{0}$ is the space permeability and $B_{\text {lobe }}$ is the lobe magnetic field $(\sim 10 \mathrm{nT})$ [cf. Kivelson and Khurana, 2002]. These parameters result in $j_{\| 1}(h) \sim 0.2 \mathrm{nA} / \mathrm{m}^{2}$. If this value is simply mapped to the ionosphere with the scaling factor of $B_{i} / B(h) \sim 10^{5}$, where $B_{i}$ is the magnetic field at the ionosphere, $j_{\| 1} \sim 20 \mu \mathrm{A} / \mathrm{m}^{2}$. However, this would be an overestimate since the assumption that $\left|\nabla B \cdot \mathbf{j}_{\perp}\right| \sim|\nabla B| \mathbf{j}_{\perp} \mid$, which was applied implicitly in the above estimation, is not true (i.e., $\mathbf{j}_{\perp}$ is not ideally parallel/anti-parallel to $\nabla B$ at flanks, due to the $\mathbf{j}_{\perp}$ component along the flanks; see Figure 5). In addition, $D_{B}$ is not well constrained from observations and larger $D_{B}$ results in smaller $j_{\| 1}$. Nevertheless, if $1 \%$ of the above value (i.e., $2 \mathrm{pA} / \mathrm{m}^{2}$ at the outer edge of the current sheet) reaches the ionosphere, $j_{\| 1}$ is $\sim 0.2 \mu \mathrm{A} / \mathrm{m}^{2}$ at the ionosphere and thus substantial auroral emission is expected in the form of transient arcs or spots, considering the field aligned current density of $0.1-1 \mu \mathrm{A} / \mathrm{m}^{2}$ for the main oval [e.g., Gustin et al., 2004]. The other two terms in equation (3) are estimated to be smaller than $j_{\| 1}$, although we cannot completely rule out their participation, considering the uncertainty in the estimation. Regardless of the most significant term, however, an important conclusion here is that at least one term in the right-hand side of equation (3) has a sufficient magnitude for field-aligned current which generates substantial auroral emission at the dawnside.

[31] Similarly, nightside auroral spots [Grodent et al., 2004], which appear at earlier local times than transient dawn arcs, may be a manifestation of duskside/midnight reconnection jets. They may correspond to the moderate reconnection jets as seen in Figure 4c, or it is also possible 
that they are generated by more prominent events in deep tail $\left(>150 \mathrm{R}_{J}\right)$ region, which we have not seen in the Galileo data set.

\section{Summary}

[32] We have examined the $B_{\theta}$ events listed by Vogt et al. [2010], where the magnetic north-south component significantly enhanced. We not only investigated the $B_{\theta}$ time profile but also examined the ion velocity and electron density, which are derived from energetic particle data and radio wave data, respectively. We have found that prominent dawnside events are consistent with reconnection jet fronts propagating planetward or tailward, and the former merge with corotational flow in the dawnside magnetosphere. A significant density drop after the front passage suggests lobe reconnection. On the other hand, prominent duskside events show different signatures. The flows are in the corotational direction (thus not from the tail) and a significant change in the electron density was rarely observed around the magnetic field peaks. Therefore most of the duskside events may not be directly related to tail reconnection events, although we do not exclude the possibility of duskside tail reconnection. The observed dawn-dusk asymmetry would be unique to rotation-dominant planetary magnetospheres, which retain (sub-)corotating plasma sheets. On the other hand, as is expected by analogy to the Earth's case, fieldaligned currents inferred from the observations are consistent with the intense auroral arcs or spots in the ionosphere.

[33] Acknowledgments. The authors thank A. Lagg for providing the EPD software. We are also grateful to B. Kurth for the PWS data. NASA's PDS website, from which MAG data were downloaded, is also appreciated. The authors would also like to make a cordial acknowledgment to M. Vogt for her useful event list, on which this study is based. S.K. enjoyed fruitful discussion with K. Fukazawa and T. Ogino.

\section{References}

Birmingham, T. J., and T. G. Northrop (1979), Theory of Flux Anisotropies in a Guiding Center Plasma, J. Geophys. Res., 84(A1), 41-45, doi:10.1029/JA084iA01p00041.

Cowley, S. W. H., E. J. Bunce, T. S. Stallard, and S. Miller (2003), Jupiter's polar ionospheric flows: Theoretical interpretation, Geophys. Res. Lett., 30(5), 1220, doi:10.1029/2002GL016030.

Cowley, S. W. H., I. I. Alexeev, E. S. Belenkaya, E. J. Bunce, C. E. Cottis, V. V. Kalegaev, J. D. Nichols, R. Prange, and F. J. Wilson (2005), A simple axisymmetric model of magnetosphere-ionosphere coupling currents in Jupiter's polar ionosphere, J. Geophys. Res., 110, A11209, doi:10.1029/2005JA011237.

Forman, M. A. (1970), The Compton-Getting effect for cosmic-ray particles and photons and the Lorentz-invariance of distribution functions, Planet. and Space Sci., 18(25), doi:10.1016/0032-0633(70)90064-4.

Fu, H. S., Y. V. Khotyaintsev, A. Vaivads, M. André, and S. Y. Huang (2012), Occurrence rate of earthward-propagating dipolarization fronts, Geophys. Res. Lett., 39, L10101, doi:10.1029/2012GL051784.

Fukazawa, K., T. Ogino, and R. J. Walker (2005), Dynamics of the Jovian magnetosphere for northward interplanetary magnetic field (IMF), Geophys. Res. Lett., 32, L03202, doi:10.1029/2004GL021392.

Fukazawa, K., T. Ogino, and R. J. Walker (2006), Configuration and dynamics of the Jovian magnetosphere, J. Geophys. Res., 111, A10207, doi:10.1029/2006JA011874.

Fukazawa, K., T. Ogino, and R. J. Walker (2010), A simulation study of dynamics in the distant Jovian magnetotail, J. Geophys. Res., 115 , A09219, doi:10.1029/2009JA015228.

Geiss, J., et al. (1992), Plasma composition in Jupiter's magnetosphereInitial results from the solar wind ion composition spectrometer, Science, $257,1535-1539$.
Grodent, D., J. T. Clarke, J. Kim, J. H. Waite Jr., and S. W. H. Cowley (2003), Jupiter's main auroral oval observed with HST-STIS, J. Geophys. Res., 108(A11), 1389, doi:10.1029/2003JA009921.

Grodent, D., J.-C. Gérard, J. T. Clarke, G. R. Gladstone, and J. H. Waite Jr.(2004), A possible auroral signature of a magnetotail reconnection process on Jupiter, J. Geophys. Res., 109, A05201, doi:10.1029/ 2003JA010341.

Gurnett, D. A., W. S. Kurth, R. R. Shaw, A. Roux, R. Gendrin, C. F. Kennel, F. L. Scarf, and S. D. Shawhan (1992), The Galileo plasma wave investigation, Space Sci. Rev., 60, 341-355, doi:10.1007/BF00216861.

Gustin, J., J.-C. Gerard, D. Grodent, S. W. H. Cowley, J. T. Clarke, and A. Grard (2004), Energy-flux relationship in the FUV Jovian aurora deduced from HST-STIS spectral observations, J. Geophys. Res., 109, A10205, doi:10.1029/2003JA010365.

Hasegawa, A. and T. Sato (1979), Generation of field aligned current during substorm, in Dynamics of the Magnetosphere, edited by S.-I. Akasofu, p. 529, D. Reidel, Dordrecht.

Jia, X., K. C. Hansen, T. I. Gombosi, M. G. Kivelson, G. Tóth, D. L. DeZeeuw, and A. J. Ridley (2012), Magnetospheric configuration and dynamics of Saturn's magnetosphere: A global MHD simulation, J. Geophys. Res., 117, A05225, doi:10.1029/2012JA017575.

Kasahara, S., E. A. Kronberg, N. Krupp, T. Kimura, C. Tao, S. V. Badman, A. Retinò, and M. Fujimoto (2011), Magnetic reconnection in the Jovian tail: X-line evolution and consequent plasma sheet structures, J. Geophys. Res., 116, A11219, doi:10.1029/2011JA016892.

Kivelson, M. G., K. K. Khurana, J. D. Means, C. T. Russell, and R. C. Snare (1992), The Galileo magnetic field investigation, Space Sci. Rev., 60, 357, doi:10.1007/BF00216862.

Kivelson, M. G., and K. K. Khurana (2002), Properties of the magnetic field in the Jovian magnetotail, J. Geophys. Res., 107, 1196, doi:10.1029/ 2001JA000249.

Kronberg, E. A., J. Woch, N. Krupp, A. Lagg, K. K. Khurana, and K.-H. Glassmeier (2005), Mass release at Jupiter: Substorm-like processes in the Jovian magnetotail, J. Geophys. Res., 110, A03211, doi:10.1029/ 2004JA010777.

Kronberg, E. A., K.-H. Glassmeier, J. Woch, N. Krupp, A. Lagg, and M. K. Dougherty (2007), A possible intrinsic mechanism for the quasi-periodic dynamics of the Jovian magnetosphere, J. Geophys. Res., 112, A05203, doi:10.1029/2006JA011994.

Kronberg, E. A., J. Woch, N. Krupp, and A. Lagg (2008), Mass release process in the Jovian magnetosphere: Statistics on particle burst parameters, J. Geophys. Res., 113, A10202, doi:10.1029/2008JA013332.

Kronberg, E., S. Kasahara, N. Krupp, and J. Woch (2012), Field-aligned beams and reconnection in the Jovian magnetotail, Icarus, 217(1), 55-65.

Krupp, N., A. Lagg, S. Livi, B. Wilken, J. Woch, E. C. Roelof, and D. J. Williams (2001), Global flows of energetic ions in Jupiter's equatorial plane: First-order approximation, J. Geophys. Res., 106(A11), 26,017-26,032, doi:10.1029/2000JA900138.

Kurth, W. S. (1992), Continuum radiation in planetary magnetospheres, in Planetary Radio Emissions III, edited by H. O. Rucker, S. J. Bauer, and M. L. Kaiser, p. 329, Austrian Academy of Science Press, Vienna.

Lui, A. T. Y. (1996), Current disruption in the Earth's magnetosphere: Observations and models, J. Geophys. Res., 101(A6), 13,067-13,088, doi:10.1029/96JA00079.

Lyons, L. R., Y. Nishimura, X. Xing, A. Runov, V. Angelopoulos, E. Donovan, and T. Kikuchi (2012), Coupling of dipolarization front flow bursts to substorm expansion phase phenomena within the magnetosphere and ionosphere, J. Geophys. Res., 117, A02212, doi:10.1029/ 2011JA017265.

Nishida, A. (1983), Reconnection in the Jovian magnetosphere, Geophys. Res. Lett., 10(6), 451-454, doi:10.1029/GL010i006p00451.

Ogino, T., R. J. Walker, and M. G. Kivelson (1998), A global magnetohydrodynamic simulation of the Jovian magnetosphere, J. Geophys. Res., 103(A1), 225-235, doi:10.1029/97JA02247.

Ohtani, S., M. A. Shay, and T. Mukai (2004), Temporal structure of the fast convective flow in the plasma sheet: Comparison between observations and two-fluid simulations, J. Geophys. Res., 109, A03210, doi:10.1029/ 2003JA010002.

Radioti, A., D. Grodent, J.-C. Gerard, and B. Bonfond (2010), Auroral signatures of flow bursts released during magnetotail reconnection at Jupiter, J. Geophys. Res., 115, A07214, doi:10.1029/2009JA014844.

Radioti, A., D. Grodent, J.-C. Gerard, M. F. Vogt, M. Lystrup, and B. Bonfond (2011), Nightside reconnection at Jupiter: Auroral and magnetic field observations from 26 July 1998, J. Geophys. Res., 116, A03221, doi:10.1029/2010JA016200.

Runov, A., V. Angelopoulos, M. I. Sitnov, V. A. Sergeev, J. Bonnell, J. P. McFadden, D. Larson, K.-H. Glassmeier, and U. Auster (2009), THEMIS observations of an earthward-propagating dipolarization front, Geophys. Res. Lett., 36, L14106, doi:10.1029/2009GL038980. 


\section{KASAHARA ET AL.: JOVIAN TAIL EVENTS}

Runov, A., V. Angelopoulos, X.-Z. Zhou, X.-J. Zhang, S. Li, F. Plaschke, and J. Bonnell (2011), A THEMIS multicase study of dipolarization fronts in the magnetotail plasma sheet, J. Geophys. Res., 116, A05216, doi:10.1029/2010JA016316.

Russell, C. T., K. K. Khurana, D. E. Huddleston, and M. G. Kivelson (1998), Localized reconnection in the near Jovian magnetotail, Science, 280(5366), 1061-1064, doi:10.1126/science.280.5366.1061.

Sergeev, V., V. Angelopoulos, S. Apatenkov, J. Bonnell, R. Ergun, R. Nakamura, J. McFadden, D. Larson, and A. Runov (2009), Kinetic structure of the sharp injection/dipolarization front in the flow-braking region, Geophys. Res. Lett., 36, L21105, doi:10.1029/2009GL040658.

Sitnov, M. I., M. Swisdak, and A. V. Divin (2009), Dipolarization fronts as a signature of transient reconnection in the magnetotail, J. Geophys. Res., 114, A04202, doi:10.1029/2008JA013980.

Vogt, M. F., M. G. Kivelson, K. K. Khurana, S. P. Joy, and R. J. Walker (2010), Reconnection and flows in the Jovian magnetotail as inferred from magnetometer observations, J. Geophys. Res., 115, A06219, doi:10.1029/2009JA015098.
Waldrop, L. S., T. A. Fritz, M. G. Kivelson, K. Khurana, N. Krupp, and A. Lagg (2005), Jovian plasma sheet morphology: Particle and field observations by the Galileo spacecraft, Planet. Space Sci., 53, 681-692, doi:10.1016/j.pss.2004.11.003.

Walker, R. J., T. Ogino, and M. G. Kivelson (2001), Magnetohydrodynamic simulations of the effects of the solar wind on the Jovian magnetosphere, Planet. Space Sci., 49, 237-245.

Williams, D. J., R. W. McEntire, S. Jaskulek, and B. Wilken (1992), The Galileo energetic particles detector, Space Sci. Rev., 60, 385-412, doi:10.1007/BF00216863.

Woch, J., N. Krupp, and A. Lagg (2002), Particle bursts in the Jovian magnetosphere: Evidence for a near-Jupiter neutral line, Geophys. Res. Lett., 29(7), 1138, doi:10.1029/2001GL014080.

Zhang, X.-J., V. Angelopoulos, A. Runov, X.-Z. Zhou, J. Bonnell, J. P. McFadden, D. Larson, and U. Auster (2011), Current carriers near dipolarization fronts in the magnetotail: A THEMIS event study, J. Geophys. Res., 116, A00I20, doi:10.1029/2010JA015885. 